\title{
Calendario 2018 - Eventi formativi FeDerSerD
}

\section{CONGRESSO NAZIONALE}

IL RAGGIUNGIMENTO DEGLI OUTCOME NELLA CLINICA DELLE DIPENDENZE: METODI E STRATEGIE PER L'ORGANIZZAZIONE DEI SERVIZI PER LE DIPENDENZE

Sede e Data: ROMA - OTTOBRE 2018

Il Congresso si pone l'obiettivo di definire, in chiave moderna ed innovativa, gli outcome clinici dei Ser.D., partendo da quello che rappresenta il forte mandato istituzionale dei Servizi e che è chiaramente definito dalle norme sia nazionali che regionali.

\section{CONGRESSI MACRO-REGIONALI}

ITRATTAMENTI FARMACOLOGICI NEL PROCESSO TERAPEUTICO-RIABILITATIVO DEL CONSUMATORE DI SOSTANZE: PROSPETTIVE ED INNOVAZIONE NEI NUOVI SCENARI E STILI DI CONSUMO

Sede e Data: FIRENZE - Centro Congressi Hotel LONDRA - 23 MARZO 2018

Il Convegno si pone l'obiettivo di presentare come i trattamenti farmacologici delle dipendenze si sono evoluti negli ultimi anni ma soprattutto di declinare i nuovi significati che i trattamenti possono avere nei processi di presa in carico del consumatore di sostanze, con e senza co-morbilità, e gli impatti che i diversi trattamenti farmacologici possono avere sui diversi stili e modalità di consumo.

LA COMORBILITÀ NELLA CLINICA DELLA DIPENDENZA: RUOLO E CENTRALITÀ DEI SER.D. NELLA PRESA IN CARICO DEL CONSUMATORE PROBLEMATICO

Sede e Data: MILANO - Centro Congressi Hotel MICHELANGELO - 18 MAGGIO 2018

Il Convegno affronterà il tema delle co-morbilità nella clinica delle dipendenze: intendendo sia quelle psichiatriche (includendo tutte le fasi del ciclo della vita) che quelle internistiche-infettivologiche.

LA PRESA IN CARICO PRECOCE DEL CONSUMATORE DI SOSTANZE: MODELLI, STRATEGIE PER UNA NUOVA ORGANIZZAZIONE DEI SERVIZI PER LE DIPENDENZE

Sede e Data: NAPOLI - GIUGNO 2018

Il Convegno affronterà il tema della presa in carico precoce del consumatore di sostanze cercando di delineare modelli e strategie operative da applicare nell'organizzazione dei Servizi. In particolare, il convegno, partendo da una analisi dei bisogni e dell'evoluzione dei consumi, proverà ad ipotizzare delle nuove modalità operative in grado di intercettare i nuovi consumatori, come quelle basate sugli outreach programs e/o le azioni di prevenzione ed intercettazione sul territorio di tipo "decentrato".

L'ALCOLISTA NEL SERVIZI PER LE DIPENDENZE: COME SCEGLIERE I PERCORSI ED I TRATTAMENTI IN BASE ALLE EVIDENZE SCIENTIFICHE

Sede e Data: NUORO (SARDEGNA) - SETTEMBRE 2018

Il convegno affronterà il tema estremamente importante, ma nello stesso tempo a volte negletto, del ruolo dei Ser.D. nella presa in carico del paziente alcolista. In particolare, il convegno approfondirà la scelta dei percorsi e dei trattamenti per il paziente alcolista, affrontando il tema del ruolo dei trattamenti farmacologici nel processo terapeutico-riabiliativo alla luce delle più moderne evidenze scientifiche.

LA TERAPIA OPPIACEA PER IL TRATTAMENTO DEL DOLORE: IL RUOLO DEI SERVIZI PER LE DIPENDENZE PER IL MIGLIORAMENTO DELL'EFFICACIA E DELLA SICUREZZA DEI TRATTAMENTI

Sede e Data: PALERMO (SICILIA) - NOVEMBRE 2018

Il Convegno affronterà il tema dell'incremento, anche nel nostro Paese, delle forme di abuso e misuso dei farmaci per il trattamento del dolore e come i Servizi per le Dipendenze possono da una parte ridurre nella popolazione generale trattata con i farmaci oppiacei per il dolore eventuali forme di uso problematico e dall'altra come possono sviluppare strategie efficaci per la presa in carico di soggetti con forme di dipendenza da questa classe di farmaci. 